\title{
A NOTE ON DILWORTH'S EMBEDDING THEOREM
}

\author{
WILLIAM T. TROTTER, JR.
}

ABSTRACT. The dimension of a poset $X$ is the smallest positive integer $t$ for which there exists an embedding of $X$ in the cartesian product of $t$ chains. R. P. Dilworth proved that the dimension of a distributive lattice $L=\underline{2}^{X}$ is the width of $X$. In this paper we derive an analogous result for embedding distributive lattices in the cartesian product of chains of bounded length. We prove that for each $k \geq 2$, the smallest positive integer $t$ for which the distributive lattice $L=\underline{2}^{X}$ can be embedded in the cartesian product of $t$ chains each of length $k$ equals the smallest positive integer $t$ for which there exists a partition $X=C_{1} \cup C_{2} \cup \cdots \cup C_{t}$ where each $C_{i}$ is a chain of at most $k-1$ points.

1. Preliminaries. A poset consists of a pair $(X, P)$ where $X$ is a set and $P$ is a reflexive, antisymmetric, and transitive relation on $X$. The notations $(x, y) \in P$ and $x \leq y$ in $P$ are used interchangeably. If $x$ and $y$ are distinct points in $X$ and neither $(x, y)$ nor $(y, x)$ is in $P$, then we say $x$ and $y$ are incomparable and write $x l y$. For convenience we will frequently use a single symbol to denote a poset. If $X$ and $Y$ are isomorphic posets, then we write $X=Y$ and if $X$ is isomorphic to a subposet of $Y$, then we write $X \subseteq Y$. The dual of a poset $X$, denoted $\hat{X}$, is the poset on the same set with $x \leq y$ in $\hat{X}$ iff $y \leq x$ in $X$.

If $(X, P)$ and $(Y, Q)$ are posets, their free sum, denoted $X+Y$, is the poset $(X \dot{\cup} Y, P \dot{U} Q)$ where $\dot{U}$ denotes disjoint union. Their cartesian product $X \times Y$ is the poset $(X \times Y, S)$ where $S=\{((x, y),(z, w)): x \leq z$ in $X$ and $y \leq w$ in $Y\}$. The cartesian product of $n$ copies of $X$ is denoted $X^{n}$. The join of $(X, P)$ and $(Y, Q)$, denoted $X \oplus Y$, is the poset $(X \dot{\cup} Y, P \cup Q$ $\cup X \times Y)$. A function $f: Y \rightarrow X$ is order preserving iff $y \leq w$ in $Y$ implies $f(y) \leq f(w)$ in $X$. The cardinal power of $X$ and $Y$, denoted $X^{Y}$, is the poset consisting of all ordering preserving functions from $Y$ to $X$ with $f \leq g$ in $X^{Y}$ iff $f(y) \leq g(y)$ in $X$ for every $y \in Y$.

A poset $C$ for which $x, y \in C$ imply $x \leq y$ or $y \leq x$ is called a chain. We denote the $n$ element chain $0<1<2<\cdots<n-1$ by $\underline{n}$. A chain $(X, L)$ is said to be linear extension of $(X, P)$ when $P \subseteq L$. We also say $L$ is a linear extension of $P$. By a theorem of Szpilrajn [12], if $\mathcal{C}$ denotes the collection of all linear extensions of $P$, then $\cap \mathcal{C}=P$.

Presented to the Society, November 8, 1974; received by the editors July 5, 1974. AMS (MOS) subject classifications (1970). Primary 06A10, 06A35.

Key words and phrases. Distributive lattice, dimension of a partitially ordered set, matching. 
$A$ poset $A$ for which $x, y \in A$ and $x \neq y$ imply $x l y$ is called an antichain. We denote an element antichain by $\bar{n}$. The width of a poset $X$, denoted $W(X)$, is the number of elements in a maximum antichain in $X$.

The justification for the exponential notation for the cardinal power of posets is given by the following property (see [2] for details).

Fact 1. $X^{\mathrm{Y}+Z}=X^{Y} \times X^{Z}$.

In this paper we are concerned primarily with cardinal powers of the form $\underline{2}^{X}$. For such posets, we have

Fact 2. $\underline{2}^{n}-=\underline{n+1}$ and $\underline{2}^{\bar{n}}=\underline{2}^{n}$.

If $(X, P)$ and $(Y, Q)$ are posets, $X=Y$, and $P \subseteq Q$, then it is easy to see that $\underline{2}^{Y} \subseteq \underline{2}^{X}$. In fact a stronger result holds.

Lemma 1. Let $(X, P)$ and $(Y, Q)$ be posets, $Y \subseteq X$, and $P \cap(Y \times Y)$ $\subseteq Q$. Then $\underline{2}^{Y} \subseteq \underline{2}^{X}$.

Proof. Define a function $F: \underline{2}^{Y} \rightarrow 2^{X}$ by $F(f)(x)=f(x)$ if $x \in Y, F(f)(x)$ $=0$ if $x \in X-Y$ and there exists $y \in Y$ such that $y>x$ in $X$ and $f(y)=$ 0 , and $F(f)(x)=1$ otherwise. It is straightforward to verity that $F$ is an embedding.

2. Introduction. Dushnik and Miller [5] defined the dimension of a poset $X$, denoted $\operatorname{Dim} X$, as the smallest positive integer $t$ for which there exist $t$ linear extensions $L_{1}, L_{2}, \ldots, L_{t}$ of the partial ordering $P$ on $X$ such that $L_{1} \cap L_{2} \cap \ldots \cap L_{t}=P$. Ore [9] gave an equivalent definition of $\operatorname{Dim} X$ as the smallest positive integer $t$ for which $X \subseteq C_{1} \times C_{2} \times \cdots \times C_{t}$ where each $C_{i}$ is a chain.

A very important example of a poset is a distributive lattice for which we have the following well-known representation theorem: $A$ poset $M$ is a distributive lattice iff $M=\underline{2}^{X}$ for some poset $X$. In 1950, R. P. Dilworth [4] published the following theorem giving the dimension of a distributive lattice.

Theorem 1. $\operatorname{Dim} 2^{X}=W(X)$.

In order to prove Theorem 1, Dilworth derived his famous decomposition theorem.

Theorem 2. If $X$ is a poset and $W(X)=n$, then the point set $X$ can be partitioned into $n$ subsets $C_{1}, C_{2}, \ldots, C_{n}$ such that the subposet determined by each $C_{i}$ is a chain.

Compact proofs of Theorem 2 appear in [10] and [15] and Theorem 1 is also discussed in [11].

In this paper we generalize the concept of dimension for posets to obtain an extension of Theorem 1. For an integer $k \geq 2$, we define the $k$-dimension of a poset $X$, denoted $\operatorname{Dim}_{k} X$ as the smallest positive integer $t$ for which $X \subseteq \underline{k}^{t}$. 
3. Some elementary inequalities. In [13], the inequality $\operatorname{Dim}_{2} X \leq|X|$ for all $X$ is established and the family of posets for which equality holds is determined. In [14], the inequalities $\operatorname{Dim}_{3} X \leq\{|X| / 2\}$ for $|X| \geq 5$ and $\operatorname{Dim}_{4} X \leq[|X| / 2]$ for $|X| \geq 6$ are established. Hiraguchi [6] proved that $\operatorname{Dim} X \leq[|X| / 2]$ for $|X| \geq 4$ and Bogart and Trotter [3] and Kimble [8] determined the collection of all posets for which equality holds.

Clearly $\operatorname{Dim} X \leq \operatorname{Dim}_{k} X$ and since $\underline{k}^{t} \subseteq \underline{k+1}{ }^{t}$, we have $\operatorname{Dim}_{k+1} X \leq$ $\operatorname{Dim}_{k} X$. Since there are $k^{t}$. points in $\underline{k}^{t}$, we have $\operatorname{Dim}_{k} X \geq \log _{k}|X|$ and since the longest chain in $\underline{k}^{t}$ has length $(k-1) t+1$, we conclude $\operatorname{Dim}_{k} \underline{n}$ $=\{(n-1) /(k-1)\}$. It is also easy to compute $\operatorname{Dim}_{k} \underline{n}$ by the methods compiled by Katona [6].

Theorem 3. $\operatorname{Dim}_{k} X \leq 2 \operatorname{Dim}_{k+1} X$.

Proof. Suppose $\operatorname{Dim}_{k+1} X=t$ and let $f: X \rightarrow \underline{k+1^{t}}$ be an embedding. Define $g: X \rightarrow \underline{k}^{2 t}$ by:

$$
g(x)(i)= \begin{cases}f(x)(i)-1 & \text { when } f(x)(i)>0 \text { and } i \leq t, \\ 0 & \text { when } f(x)(i)=0 \text { and } i \leq t, \\ f(x)(i) & \text { when } f(x)(i)<k \text { and } i>t \\ k-1 & \text { when } f(x)(i)=k \text { and } i>t .\end{cases}
$$

It follows easily that $g$ is an embedding and thus $\operatorname{Dim}_{k} X \leq 2 t$.

In order to determine whether or not the inequality of Theorem 3 is best possible, we need the following generalization of a well-known property (see [2, problem 7, p. 101]) of dimension which we state without proof.

Fact 4. If $X$ and $Y$ are posets, then $\operatorname{Dim}_{k} X \times Y \leq \operatorname{Dim}_{k} X+\operatorname{Dim}_{k} Y$. If $X$ and $Y$ have distinct greatest and least elements, then equality holds.

Since $\operatorname{Dim}_{k} \underline{k+1}=2$ and $\operatorname{Dim}_{k+1} \underline{k+1}=1$, it follows from Fact 4 that $\operatorname{Dim}_{k} \underline{k+1}^{t}=2 t$ while $\operatorname{Dim}_{k+1} \underline{k+1}^{t}=t$ for all $t \geq 1$.

4. Dilworth's embedding theorem. A short proof of Dilworth's embedding theorem (Theorem 1) is given here for the sake of completeness. We assume Theorem 2.

To show that $\operatorname{Dim} \underline{2}^{X} \leq W(X)$, let $|X|=m, W(X)=n$, and $X=C_{1} \cup$ $C_{2} \cup \ldots \cup C_{n}$ be a decomposition into chains. It follows that

$$
\underline{2}^{X} \subseteq \underline{2}^{C_{1}+C_{2}+\cdots+C_{n}}=\underline{2}^{C}{ }^{1} \times \underline{2}^{C} \times \cdots \times \underline{2}^{C} \underline{n} \subseteq \underline{m+1} \underline{1}^{n}
$$

and thus $\operatorname{Dim} \underline{2}^{X} \leq n$.

On the other hand if $A$ is an antichain of $X$ with $|A|=n$, then $\underline{2}^{n}=$ $\underline{2}^{A} \subseteq \underline{2}^{X}$ and we conclude that $\operatorname{Dim} \underline{2}^{X} \geq \operatorname{Dim} \underline{2}^{n}=n$.

The reader is invited to compare this argument with the proof of Theorem 3 in [13]. 
5. Some additional inequalities. For a poset $X$ and an integer $m \geq 1$, let $P_{m}(X)$ be the smallest positive integer $t$ for which there exists a partition of the point set of $X$ of the form $X=C_{1} \cup C_{2} \cup \cdots \cup C_{t}$ where the subposet determined by each $C_{i}$ is a chain with $\left|C_{i}\right| \leq m$. The first half of the argument given in the preceding section allows us to conclude that $\operatorname{Dim}_{k} \underline{2}^{X} \leq P_{k-1}(X)$.

Now every poset $Y$ can be written as the free sum $Y=Y_{1}+Y_{2}+\cdots+$ $Y_{r}$ of its components. For a poset $Y$ with components $Y_{1}, Y_{2}, \ldots, Y_{r}$ and an integer $m \geq 1$, we then define $S_{m}(Y)=\Sigma_{i=1}^{r}\left\{\left|Y_{i}\right| / m\right\}$. To provide a generalization of the concept of width, we define $W_{m}(X)=\max \left\{S_{m}(Y): Y \subseteq X\right\}$. Dilworth's decomposition theorem can then be restated in the following form.

Theorem 4. For every poset $X$, there exists an integer $m_{0}$ such that $m \geq m_{0}$ implies $P_{m}(X)=W_{m}(X)$.

To see the connection between these definitions and Dilworth's embedding theorem we observe that the following result holds.

Theorem 5. For every poset $X$ and every integer $k \geq 2, W_{k-1}(X) \leqq$ $\operatorname{Dim}_{k} \underline{2}^{X} \leq P_{k-1}(X)$.

Proof. Choose a subposet $Y \subseteq X$ with $W_{k-1}(X)=S_{k-1}(Y)$; let the components of $Y$ be $Y_{1}, Y_{2}, \ldots, Y_{r}$ and for each $i \leqq r$ let $C_{i}$ be a linear extension of $Y_{i}$. If follows that

$$
\begin{aligned}
& \underline{2}^{C^{1}} \times \underline{2}^{C} \times \cdots \times \underline{2}^{C} \subseteq \underline{2}^{Y^{1}} \times \underline{2}^{Y^{2}} \times \cdots \times \underline{2}^{Y_{r}} \\
& =\underline{2}^{Y_{1}+Y_{2}+\cdots+Y_{r}}=\underline{2}^{Y} \subseteq \underline{2}^{X}
\end{aligned}
$$

and therefore

$$
\begin{aligned}
& \operatorname{Dim}_{k}\left(\underline{2}^{C}{ }^{1} \times 2^{C}{ }^{2} \times \cdots \times \underline{2}^{C} r\right) \leq \operatorname{Dim}_{k} \underline{2}^{X} . \\
& \operatorname{Dim}_{k}\left(\underline{2}^{C}{ }^{1} \times \underline{2}^{C_{2}} \times \cdots \times \underline{2}^{C}\right)=\sum_{i=1}^{r}\left\{\left|C_{i}\right| /(k-1)\right\}=\sum_{i=1}^{r}\left\{\left|Y_{i}\right| /(k-1)\right\} \\
& =S_{k-1}(Y)=W_{k-1}(X) \text {. }
\end{aligned}
$$

For $m=1, W_{1}(X)=P_{1}(X)=|X|$ for all $X$. It is also true that $W_{2}(X)=$ $P_{2}(X)$ for all $X$; in fact a more general result holds which we outline here. For a graph $H$ with components $H_{1}, H_{2}, \ldots, H_{r}$ let $S_{m}(H)=\sum_{i=1}^{r}\left\{\left|H_{i}\right| / m\right\}$. For a graph $G$, let $W_{m}(G)=\max \left\{S_{m}(H): H\right.$ is an induced subgraph of $\left.G\right\}$. Also let $P_{m}(G)$ be the smallest positive integer $n$ for which there exists a partition of the vertex set of $G$ into $n$ subsets so that the induced subgraph spanned by each subset is a complete graph on at most $m$ vertices. 
For a poset $X$ the comparability graph of $X$, denoted $G_{X}$, is the graph whose vertex set is the point set of $X$ with distinct points $x, y \in X$ adjacent in $G_{X}$ iff $x<y$ or $y<x$ in $X$. Clearly $P_{m}(X)=P_{m}\left(G_{X}\right)$ and $W_{m}(X)=$ $W_{m}\left(G_{X}\right)$.

Theorem 6. $W_{2}(G)=P_{2}(G)$ for all graphs.

Proof. We assume Hall's matching theorem for graphs and then proceed by induction on $|X|$. Now suppose $G$ is a graph with $W_{2}(G)=t$ and let $H$ be a subgraph of $G$ with components $H_{1}, H_{2}, \ldots, H_{r}$ so that $W_{2}(G)=W_{2}(H)$ $=\sum_{i=1}^{r}\left\{\left|H_{i}\right| / 2\right\}=t$. We further assume that $H$ is chosen so that $r$ is maximal and $|H|$ is minimal. Thus $W_{2}\left(H_{i}-x\right)<W_{2}\left(H_{i}\right)$ for every $i \leq r$ and every $x$ $\in H_{i}$ and we may assume that $H \neq X$.

Now construct a bipartite graph $(X, Y)$ with $X=\left\{v_{1}, v_{2}, \ldots, v_{r}\right\}$ and $Y=G-H$. A vertex $y \in Y$ is adjacent to $v_{i}$ in $(X, Y)$ iff $y$ is adjacent to at least one vertex of $H_{i}$ in $G$.

By Hall's matching theorem, there exists a matching of $Y$ into $X$ for if $Y^{1} \subseteq Y, X^{1}=\left\{v \in X: v \perp y\right.$ for some $\left.y \in Y^{1}\right\}$, and $\left|X^{1}\right|<\left|Y^{1}\right|$, then $W_{2}\left(H \cup Y^{1}\right)>W_{2}(H)$.

We then assume that the elements of $Y$ are labeled so that $Y=\left\{y_{1}\right.$, $\left.y_{2}, \ldots, y_{s}\right\}, s \leq r$, and $y_{i} \perp H_{i}$ in $(X, Y)$ for each $i \leq s$. We then choose vertices $a_{1}, a_{2}, \ldots, a_{s}$ from $H_{1}, H_{2}, \ldots, H_{s}$ so that $y_{i} \perp a_{i}$ in $G$ for each $i \leq s$. From the inductive hypothesis, we conclude that for each $i \leq s$, the subgraph $H_{i}-a_{i}$ can be partitioned into $W_{2}\left(H_{i}\right)-1$ complete subgraphs each of at most two vertices.

Since $s \geq 1$, we may partition for each $i$ with $s+1 \leq i \leq r$, the subgraph $H_{i}$ into $W_{2}\left(H_{i}\right)$ complete subgraphs of at most two vertices. When combined with $\left\{y_{1}, a_{1}\right\},\left\{y_{2}, a_{2}\right\}, \ldots,\left\{y_{s}, a_{s}\right\}$, the construction produces a partition of $G$ into $W_{2}(G)$ complete subgraphs of at most two vertices.

Anderson [1] uses a similar argument to give an elementary proof of Tutte's factor theorem from Hall's matching theorem.

It is not true that $W_{3}(G)=P_{3}(G)$ for all graphs. An example of a poset $X$ for which $W_{3}(X)<P_{3}(X)$ is $(\underline{3}+\underline{3})+\overline{3}$.

6. An extension of Dilworth's embedding theorem. In this section we consider the structure of $\underline{2}^{X}$ in more detail in order to make an exact computation of $\operatorname{Dim}_{k} \underline{2}^{X}$.

Theorem 7. $\operatorname{Dim}_{k} \underline{2}^{X}=P_{k-1}(X)$ for all $X$.

Proof. Suppose $\operatorname{Dim}_{k} \underline{2}^{X}=t$ and let $F: \underline{2}^{X} \rightarrow \underline{k}^{t}$ be an embedding. For each $x \in X$ let $f_{x}: X \rightarrow \underline{2}$ be defined by $f_{x}(y)=0$ if $y \leq x$ in $X$ and $f_{x}(y)$ $=1$ otherwise. It follows that $f_{x} \in \underline{2}^{X}$ for every $x \in X$ and $f_{x}<f_{y}$ in $\underline{2}^{X}$ 
iff $x>y$ in $X$, i.e. the map $g: \hat{X} \rightarrow \underline{2}^{X}$ defined by $g(x)=f_{x}$ is an embedding.

For each $i \leq t$ let $X_{i}=\left\{x \in X: y<x\right.$ or $y I x$ implies $\left.F\left(f_{x}\right)(i)<F\left(f_{y}\right)(i)\right\}$. Then each $X_{i}$ is a chain in $X$ with $\left|X_{i}\right| \leq k$. Furthermore if $\left|X_{i}\right|=k$, then the least element in $X_{i}$ is also the least element in $X$.

We now show that $X=X_{1} \cup X_{2} \cup \ldots \cup X_{t}$. Suppose on the contrary that there exists $x \in X$ with $x \notin X_{1} \cup X_{2} \cup \ldots \cup X_{i}$. Then for each $i \leq t$, there exists a point $y \in X$ with $y \notin X$ but $F\left(f_{x}\right)(i) \geq F\left(f_{y}\right)(i)$. Let $\mathcal{C}$ be the collection of all subsets $A \subseteq X$ such that (1) $a \in A$ implies $a \not x x$ and (2) for every $i \leq t$, there exists $a \in A$ with $F\left(f_{x}\right)(i) \geq F\left(f_{a}\right)(i)$. Now among the sets in $\mathcal{C}$, choose one set say $A_{0}$ with $\left|A_{0}\right|$ minimum. It follows that $A_{0}$ is an antichain and $\left|A_{0}\right| \geq 2$. Now define a function $f_{0}: X \rightarrow \underline{2}$ by $f_{0}(y)=0$ if $y \leq a$ for some $a \in A_{0}$ and $f_{0}(y)=1$ otherwise. It follows that $f_{0} \in \underline{2}^{X}$ and $f_{0}<f_{a}$ in $\underline{2}^{X}$ for every $a \in A_{0}$. Furthermore $f_{0} \underline{\lfloor} f_{x}$ in $\underline{2}^{X}$ since $f_{0}(x)=1$ and $f_{x}(x)=0$. Since $F$ is an embedding of $\underline{2}^{X}$ in $\underline{k}^{t}$, there exist $i \leq t$ with $F\left(f_{0}\right)(i)>F\left(f_{x}\right)(i)$ and thus $F\left(f_{a}\right)(i)>F\left(f_{x}\right)(i)$ for every $a \in A_{0}$. The contradiction shows that $X=X_{1} \cup X_{2} \cup \cdots \cup X_{t}$.

If $X$ has no least element, then $\left|X_{i}\right| \leq k-1$ for all $i \leq t$ and thus $P_{k-1}(X) \leq t$. If $X$ has a least element $x$, remove $x$ from each chain in which it appears and let the resulting chains be $Y_{1}, Y_{2}, \ldots, Y_{t}$. If $\left|Y_{i}\right| \leq$ $k-2$ for some $i \leq t$, then we conclude that $P_{k-1}(X) \leq t$ since

$$
X=Y_{1} \cup Y_{2} \cup \cdots \cup\left(Y_{i} \cup\{x\}\right) \cup \cdots \cup Y_{i} .
$$

If $\left|Y_{i}\right|=k-1$ for every $i \leq t$, then $F\left(f_{x}\right)(i)=k-1$ for every $i \leq t$. Define $h: X \rightarrow \underline{2}$ by $h(y)=1$ for all $y \in X$. Then $h>f_{x}$ in $\underline{2}^{X}$ but $F\left(f_{x}\right) \geq F(h)$ in $\underline{k}^{t}$. The contradiction completes the proof.

\section{B IB LIOGR A PHY}

1. I. Anderson, Perfect matching of a graph, J. Combinational Theory Ser. B 10 (1971), 183-186. MR 43 \#1853.

2. G. Birkhoff, Lattice theory, 3rd ed., Amer. Math. Soc. Colloq. Publ., vol. 25, Amer. Math. Soc., Providence, R. I., 1967. MR 37 \#2638.

3. K. P. Bogart and W. T. Trotter, Maximal dimensional partially ordered sets.

II. Characterization of $2 n$-element posets with dimen sion $n$, Discrete Math. 5

(1973), 33-43. MR 47 \#6563.

4. R. P. Dilworth, A decomposition theorem for partially ordered sets, Ann. of Math. (2) 51 (1950), 161-166. MR 11, 309.

5. B. Dushnik and E. W. Miller, Partially ordered sets, Amer. J. Math. 63 (1974), 600-610. MR 3, 73.

6. T. Hiraguchi, On the dimension of orders, Sci. Rep. Kanazawa Univ. 4 (1955), no. 1,1-20. MR 17, 1045; 19, 1431.

7. Gyula Katona, A generalization of some generalizations of Sperner's theorem, J. Combinatorial Theory Ser. B 12 (1972), 72-81。 MR 44 \#2620.

8. R. Kimble, Extremal problems in dimension theory for partially ordered sets, Ph. D. Thesis, M.I.T., Cambridge, Mass., 1973. 
9. O. Ore, Theory of graphs, Amer. Math. Soc. Colloq. Publ., vol. 38, Amer. Math. Soc., Providence, R. I., 1962. MR 27 \#740.

10. M. A. Perles, A proof of Dilworth's decomposition theorem for partially ordered sets, Israel J. Math. 1 (1963), 105-107. MR 29 \#5758.

11. G.-C. Rota and L. H. Harper, Matching theory, an introduction, Advances in Probability and Related Topics, vol. 1, Dekker, New York, 1971, pp. 169-215. MR 44 \#89.

12 E. Szpilrajn, Sur l'extension de l'ordre partiel, Fund. Math. 16 (1930), 386389.

13. W. T. Trotter, Embedding finite posets in cubes, Discrete Math. (to appear).

14. - A generalization of Hiraguchi's inequality for posets, J. Combinatorial Theorv Ser. A (to apdear).

15. H. Tverberg, On Dilworth's decomposition theorem for partially ordered sets, J. Combinational Theory 3 (1967), 305-306. MR 35 \#5366.

DEPARTMENT OF MATHEMATICS AND COMPUTER SCIENCE, UNIVERSITY OF SOUTH CAROLINA, COLUMBIA, SOUTH CAROLINA 29208 